\title{
ALTA Y BAJA PRESENCIA EN LA BÚSQUEDA DE REFUERZOS TANGIBLES FUERA DEL ÁMBITO ESCOLAR: DIFERENCIAS DE PERFECCIONISMO EN POBLACIÓN ECUATORIANA
}

\author{
José Manuel García-Fernández \\ Universidad de Alicante \\ josemagf@ua.es \\ Virginia Narcisa Ortega Sandoval \\ Universidad Central del Ecuador \\ virortsan@hotmail.com \\ Aitana Fernández-Sogorb \\ Universidad de Alicante \\ aitana.fernandez@ua.es \\ María Pilar Aparicio-Flores \\ Universidad de Alicante \\ pilar.aparicio@ua.es
}

Fecha de Recepción: 12 Septiembre 2019

Fecha de Admisión: 25 Septiembre 2019

\begin{abstract}
RESUMEN
El perfeccionismo muestra un carácter multidimensional cuya característica principal en los sujetos que lo poseen es establecerse estándares excesivamente altos, autoevaluándose con demasiada rigurosidad. Por este motivo, los estudios que lo abordan han enfocado su conceptualización en observar la tendencia a trastornos psicopatológicos. Por otro lado, el rechazo escolar, entre otros aspectos, muestra una de las causas cuya lógica recae en la búsqueda de obtener recompensas positivas fuera del ámbito escolar. Es decir, ese rechazo se centra en la negativa a asistir al centro educativo ya que es considerado aburrido y se prefiere estar con los amigos o realizando actividades recreativas. El objetivo del siguiente trabajo fue analizar las diferencias estadísticamente significantes de perfeccionismo con estudiantes con altos y bajos niveles de la búsqueda de refuerzos tangibles fuera del ámbito escolar. Se utilizaron el Child-Adolescent Perfectionism Scale (CAPS) para evaluar el perfeccionismo, y la School Refusal Assessment Scale-Revised for Children (SRAS-R-C) para medir el rechazo escolar. La muestra de la población seleccionada alcanzó un total de 1920 alumnos de entre $1^{0}$ y $3^{\circ}$ de Bachillerato. Los resultados arrojan diferencias en los grupos con altos y bajos niveles del IV factor del SRAS-R-C, en Perfeccionismo Socialmente Prescrito (PSP), Perfeccionismo Auto Orientado Esfuerzos (PAO-E) y Perfeccionismo Auto Orientado Crítica (PAOC), observando puntuaciones más altas en PSP $(d=.37)$, PAO-E $(d=.38)$, y PAO-C $(d=.14)$ en
\end{abstract}


aquellos estudiantes con altos niveles de rechazo escolar frente a sus iguales con niveles más bajos de rechazo escolar. En conclusión, el presente estudio colabora con un nuevo conocimiento en el ámbito científico-educativo, e incita a seguir investigando sobre el tema, teniendo en cuenta la repercusión a nivel psicológico y académico que puede contribuir en la población juvenil ecuatoriana.

Palabras clave: CAPS; SRAS-R-C; población juvenil; Ecuador; educación.

\section{ABSTRACT}

High and low presence in the search for tangible reinforcements outside the school scope: perfectionism differences in Ecuadorian population. Perfectionism shows a multidimensional character whose main characteristic in the subjects that possess it is to establish excessively high standards, self-assessing with too much rigor. For this reason, studies that address it have focused their conceptualization on observing the tendency to psychopathological disorders. On the other hand, school rejection, among other aspects, shows one of the causes whose logic lies in the pursuit of tangible reinforcement outside of school. That is, that rejection focuses on the refusal to attend the school because it is considered boring and it is preferred to be with friends or doing recreational activities. The objective of the following work was to analyze the statistically significant differences of perfectionism with students with high and low levels of the pursuit of tangible reinforcement outside of school. The Child-Adolescent Perfectionism Scale (CAPS) was used to assess perfectionism, and the School Refusal Assessment Scale-Revised for Children (SRAS-R-C) to measure school rejection. The sample of the selected population reached a total of 1920 students between $1^{\circ}$ and $3^{\circ}$ of Baccalaureate. The results show differences in the groups with high and low levels of the IV SRAS-RC factor, in Socially Prescribed Perfectionism (SPP), Self-Oriented PerfectionismStrivings (SOP-S) and Self-Oriented Perfectionism-Critical (SOP-C), observing scores higher in SPP $(d=.37)$, SOP-S $(d=.38)$, and SOP-C $(d=.14)$ in those students with high levels of school rejection compared to their peers with lower levels of refusal school. In conclusion, the present study collaborates with new knowledge in the scientific-educational field, and encourages further research on the subject, taking into account the psychological and academic impact that can contribute to the Ecuadorian youth population.

Keywords: CAPS; SRAS-R-C; youth population; Ecuador; education.

\section{INTRODUCCIÓN}

Cuando se indaga sobre la terminología del rechazo escolar surgen diferentes concepciones tales como fobia social o escolar, absentismo, deserción y trastornos de ansiedad por separación, entre otros términos.

No obstante, estos constructos, pese a su vínculo con el rechazo escolar, han sido cuestionados como variables que lo definan porque no reflejan fielmente la conducta de rechazo al ámbito educativo (Kearney y Silverman, 1990). De ahí la importancia de indagar sobre el término, el cual se observa principalmente en edades comprendidas entre 5 y 17 años de edad, y con una prevalencia entre el $1 \%$ y el $10 \%$ de los jóvenes ya en el nivel primario de la escuela (Kearney, 1995), independientemente del grupo socioeconómico y del sexo (Last y Strauss, 1990).

Los síntomas del rechazo escolar se centran en conductas de internalización o externalización (Kearney y Albano, 2000). El alumnado con rechazo escolar se caracteriza por ausentarse de manera total o parcial del horario escolar, y si lo hacen acuden a regañadientes, lo que con el tiempo aumenta la conducta desadaptativa, tales como berrinches, incumplimientos o huidas, y con problemáticas de tipo psicofisiológica, tales como ansiedad, estrés, depresión y fatiga entre otras (Bada, 2015; Egger, Costello y Angold, 2003; Heyne, King, Tong y Cooper, 2001; Kearney, 2001; McShane, Walter y Rey, 2001). De hecho, diversos estudios afirman consecuencias negativas a 
corto y largo plazo. Estas derivan, a corto plazo, en conflictos con la familia, con el ámbito académico y social y, a largo plazo, con problemáticas delincuentes, económicas al no dotarse de recursos profesionales y, en consecuencia, maritales (Buitelaar, Van Andel, Buyx y Van Strien, 1994; Flakierska, Lindstrom y Gillberg, 1988; Kearney y Albano, 2000).

Cuatro son las razones, según Kearney (2006) por las que el alumnado rechaza la escuela: 1) por evitar aquellos estímulos que provocan una sensación general de negatividad; 2) por alejarse de esas molestias que producen las actividades académicas y sociales en las que el sujeto se ve obligado a ser partícipe; 3 ) por la búsqueda de la atención de personas significativas para el sujeto, es decir, por la necesidad de sentir esa afectividad por parte de las personas más cercanas para él con la finalidad de reafirmar su autoestima; y 4) por la búsqueda de la obtención de recompensas positivas fuera de la escuela, ya que se considera el ámbito educativo como un lugar aburrido, por lo que se prefiere estar con amigos o realizando actividades creativas.

Este último factor parece ser que busca todo lo contrario a marcarse objetivos personales elevados, tales como lo hace el perfeccionismo (Flett et al., 2016). No obstante, sería conveniente realizar un estudio que establezca su conexión, o en su defecto, desconexión.

Estos logros que las personas perfeccionistas consiguen alcanzar, se consideran en muchos casos como triunfos personales, sin embargo, dentro de estos éxitos existe un colectivo con demandas excesivamente rígidas, compulsivas e incesantes al contemplar que fallan en su proceso o resultado, lo que conlleva a insatisfacciones personales (Burns, 1980; Flett y Hewitt, 2002; Frost, Marten, Lahart y Rosenblate, 1990; Hamacheck, 1978; Hewitt y Flett, 1991). Es por ello, que el perfeccionismo se trata de una variable emocional que lleva consigo características no solo educativas, sino también laborales, sociales y personales (Stoeber y Stoeber, 2009).

Según Vicent (2017), probando las propiedades psicométricas de la Child and Adolescent Perfectionism Scale (CAPS) en población infantil española, tres son las dimensiones que enmarcan el perfeccionismo: 1) Perfeccionismo Socialmente Prescrito (PSP), el cual se caracteriza por el pensamiento de que las personas más cercanas a nosotros piensan esperan que seamos perfectos; 2) Perfeccionismo Auto-Orientado Esfuerzos (PAO-E), el cual comprende los deseos por ser perfecto, por lo que se necesita un esfuerzo incesante para ello, sin llegar a conseguir la meta; y 3) Perfeccionismo Auto-Orientado Crítica (PAO-C), el cual contempla la propia crítica y el miedo a cometer algún error. Asimismo, Ortega-Sandoval (2019), replicó el estudio con población adolescente ecuatoriana, componiendo la escala con las mismas dimensiones.

\section{OBJETIVOS DE LA INVESTIGACIÓN}

El objetivo de nuestro estudio trata de analizar si existen diferencias de los tres factores que conforman el perfeccionismo en sujetos con alto o bajo rechazo escolar, concretamente con esa búsqueda de refuerzos tangibles fuera del ámbito escolar.

\section{MUESTRA Y/O PARTICIPANTES}

La muestra total alcanzada de participantes, bajo muestreo aleatorio por conglomerados, alcanzó un total de 1786 alumno/as, después de excluir 134 participantes por omisiones en sus respuestas o por no entregar el consentimiento informado. Esta muestra variaba entre los 15 y 18 años (Medad $=16.31 ; D E=1.00)$, un $49 \%$ son chicas, y estaban matriculados entre $1^{\circ}$ y $3^{\circ}$ de bachillerato en 3 distintas instituciones públicas del país de Ecuador (véase Tabla 1). 
ALTA Y BAJA PRESENCIA EN LA BÚSQUEDA DE REFUERZOS TANGIBLES FUERA DEL ÁMBITO ESCOLAR: DIFERENCIAS DE PERFECCIONISMO EN POBLACIÓN ECUATORIANA

Tabla 1.

Tabla de contingencia sexo*curso con frecuencias y porcentajes

\begin{tabular}{|c|c|c|c|c|}
\hline & & Curso & & Total \\
\hline Sexo & $1^{\circ}$ & $2^{\circ}$ & $3^{\circ}$ & \\
\hline Chicos & 366 & 308 & 236 & 910 \\
\hline$\%$ & $20.5 \%$ & $17.2 \%$ & $13.2 \%$ & $51.0 \%$ \\
\hline Chicas & 342 & 296 & 238 & 876 \\
\hline$\%$ & $19.1 \%$ & $16.6 \%$ & $13.3 \%$ & $49.0 \%$ \\
\hline Total & 708 & 604 & 474 & 1786 \\
\hline$\%$ & $39.6 \%$ & $33.8 \%$ & $26.5 \%$ & $100.0 \%$ \\
\hline
\end{tabular}

\section{METODOLOGÍA Y/O INSTRUMENTOS UTILIZADOS}

\section{Instrumentos}

School Refusal Assessment Scale-Revised for Children (SRAS-R-C; Kearney, 2002; 2006): La SRAS-R-C es una escala tipo Likert, compuesta por 24 ítems, que mide el rechazo escolar en población infantil y juvenil. Este instrumento, contiene 7 tipos de respuesta $(0=$ Nunca; $6=$ Siempre $)$ y se divide en cuatro dimensiones: 1) Evitar la afectividad negativa que provocan los estímulos o situaciones relacionadas con el ámbito escolar (e.g.: ¿Con qué frecuencia tienes sentimientos negativos hacia la escuela (por ejemplo, asustado, nervioso o triste) cuando piensas en la escuela el sábado o el domingo?); 2). Escapar de la aversión social o situaciones de evaluación (e.g.: ¿Cuántas veces tratas de no ir a la escuela poque no tienes muchos amigos allí?); 3). Búsqueda de la atención de otras personas significativas (e.g.: ¿Cuántas veces preferirías estar con tu familia más que ir a la escuela?), y 4). Búsqueda de refuerzos tangibles fuera del ámbito escolar (e.g.: Cuando no estás en la escuela durante la semana, ¿cuánto disfrutas haciendo cosas distintas (por ejemplo, estar con amigos, salir)?). Los niveles de fiabilidad fueron aceptables ( $\alpha=$ entre .70 y .87) en su adaptación española (Gonzálvez et al., 2016).

Child and Adolescent Perfectionism Scale (CAPS; Flett, Hewitt, Boucher, Davidson y Munro, 2000; Flett et al., 2016). La versión española de la CAPS (Vicent et al., 2017) es una medida de autoinforme, con 5 puntos de respuesta ( 1 = Nada cierto en mí; 5 = Muy cierto en mí) compuesta por 13 ítems estructurados en 3 factores: 1). Perfeccionismo Socialmente Prescrito (e.g.: Mis profesores esperan que mi trabajo sea perfecto); 2). Perfeccionismo Auto Orientado-Esfuerzos (e.g.: Quiero ser el mejor en todo lo que hago); y 3). Perfeccionismo Auto Orientado-Críticas (e.g.: Incluso cuando apruebo, siento que he fallado si no he tenido una de las notas más altas de la clase). Los niveles de fiabilidad fueron aceptables en todos los casos ( $\alpha=$ entre .70 y .80).

\section{Procedimiento}

Tras realizar una entrevista con los equipos directivos de cada centro, se pasó a la administración de los cuestionarios.

La entrevista tuvo como objetivo solicitar colaboración y detallar el trabajo de investigación, así como la temática.

Después de esta entrevista, se concretó una sesión de alrededor de 20 minutos donde se administraron los cuestionarios. Cabe destacar el respeto a la privacidad y, voluntariedad, y que sola- 
mente aquellos estudiantes que obtuvieron el consentimiento informado por escrito por sus progenitores o tutores legales fueron aquellos que completaron la SRAS-R-C y la CAPS.

\section{Análisis de Datos}

Para poder determinar las diferencias existentes en PSP, PA0-E y PAO-C en función del alto 0 bajo rechazo debido al cuarto factor del SRAS-R-C, se llevaron a cabo pruebas $t$ de Student. Según Cohen (1988), las puntuaciones se consideran de magnitud pequeña cuando se encuentran entre .20 y .49, de magnitud moderada cuando contemplan tamaños entre .50 y .79 , y grandes a partir de .80 .

Por otro lado, el alto o bajo grado de rechazo escolar se contempló teniendo en cuanto que para un nivel bajo el percentil hallado es igual o inferior a 25, mientras que para un nivel elevado las puntuaciones son iguales o superiores al percentil 75 .

Los análisis estadísticos se realizaron mediante el programa estadístico SPSS 22.0.

\section{RESULTADOS ALCANZADOS}

La Tabla 2 nos muestra que existen diferencias estadísticamente significativas en las tres dimensiones de perfeccionismo (PSP, PAO-E y PAO-C) en función de las altas y bajas puntuaciones del tercer factor del SRAS-R-C, búsqueda de refuerzos tangibles fuera del ámbito escolar.

Tabla 2.

Diferencias en perfeccionismo en estudiantes con altas y bajas puntuaciones en el FIV del SRAS-R-C

\begin{tabular}{cccccccccccc}
\hline Variable & \multicolumn{2}{c}{$\begin{array}{c}\text { Prueba } \\
\text { Levene }\end{array}$} & \multicolumn{2}{c}{$\begin{array}{c}\text { Altas } \\
\text { puntuaciones }\end{array}$} & \multicolumn{2}{c}{$\begin{array}{c}\text { Bajas } \\
\text { puntuaciones }\end{array}$} & \multicolumn{4}{c}{$\begin{array}{c}\text { Significación estadística y } \\
\text { magnitud diferencias }\end{array}$} \\
\hline & $F$ & $p$ & $M$ & $D E$ & $M$ & $D E$ & $t$ & $g . l$. & $p$ & $d$ \\
\hline PSP & 9.85 & .002 & 17.48 & 4.38 & 15.85 & 4.91 & 5.10 & 626.5 & $<.001$ & .37 \\
PAO-E & 18.03 & $<.001$ & 14.08 & 3.51 & 12.66 & 4.15 & 5.34 & 599.4 & $<.001$ & .38 \\
PAO-C & .05 & .815 & 12.44 & 3.70 & 11.91 & 3.74 & 2.11 & 960 & .035 & .14
\end{tabular}

Nota: FIV SRAS =Búsqueda de refuerzos tangibles fuera de la escuela; PSP =

Perfeccionismo Socialmente Prescrito; PAO-E = Perfeccionismo Auto Orientado

Esfuerzos; PAO-C = Perfeccionismo Auto Orientado Crítica

Los adolescentes participantes con alto nivel de rechazo escolar obtuvieron puntuaciones más elevadas que sus iguales con bajos niveles de rechazo escolar para las variables PSP $(d=.37)$, PA0$\mathrm{E}(d=.38)$ y PAO-C $(d=.14)$, con bajas magnitudes de diferencia en todos los casos (véase Figura 1). 
ALTA Y BAJA PRESENCIA EN LA BÚSQUEDA DE REFUERZOS TANGIBLES FUERA DEL ÁMBITO ESCOLAR: DIFERENCIAS DE PERFECCIONISMO EN POBLACIÓN ECUATORIANA

Figura 1. Diagrama de barras para las diferencias en puntuaciones de perfeccionismo en estudiantes con altas y bajas puntuaciones en el Factor IV del SRAS.

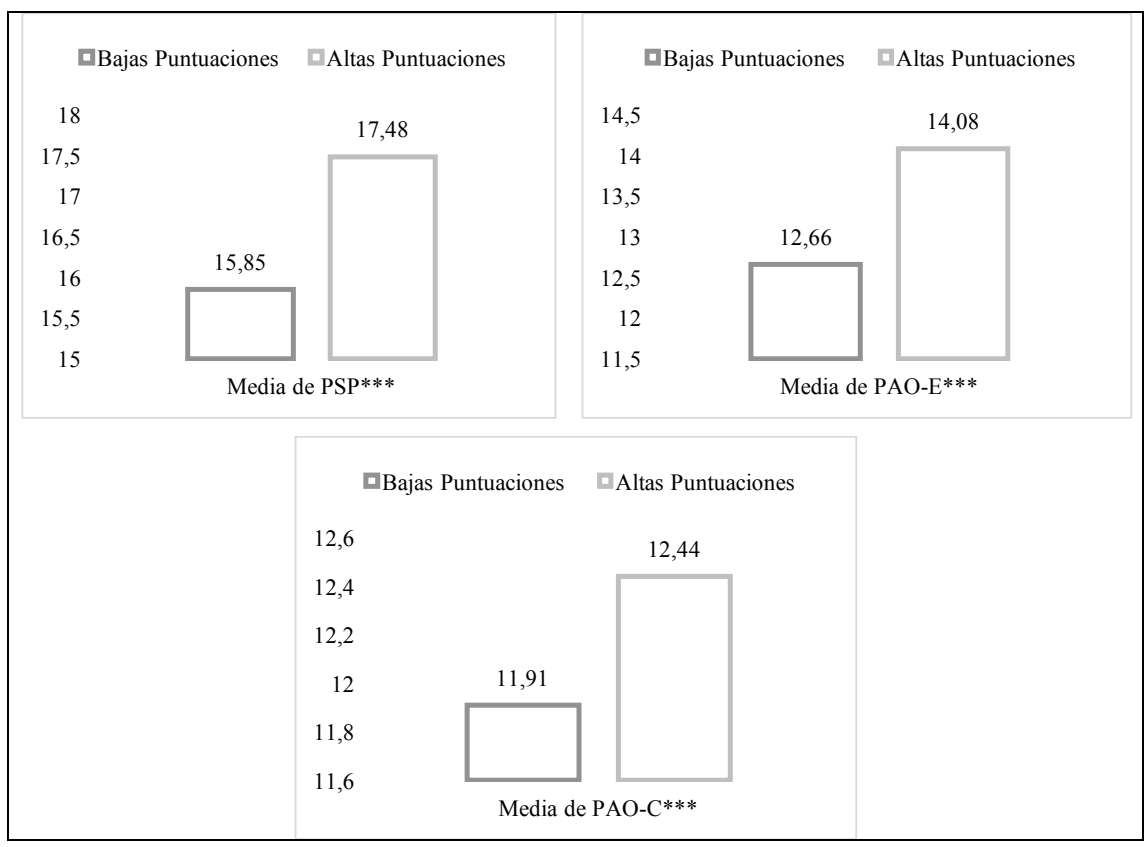

Nota: ${ }^{*}=p<.05 ;{ }^{* *}=p<.01 ;{ }^{* *}=p<.001$

\section{DISCUSIÓN}

El objetivo del estudio era analizar si existen diferencias estadísticamente significativas en las tres dimensiones de la CAPS (PSP, PAO-E y PAO-C) que marca Vicent (2017) y Ortega-Sandoval (2019), en función del cuarto factor del SRAS-R-C, la búsqueda de refuerzos tangibles fuera del ámbito escolar.

Los hallazgos marcan que aquellos estudiantes con elevadas puntuaciones en la búsqueda de refuerzos tangibles fuera del ámbito escolar puntuaron significativamente más en PSP, PAO-E y PAO-C que aquellos adolescentes con bajas puntuaciones en el factor IV del SRAS-R-C. Todas las diferencias fueron de pequeña magnitud.

Es importante tener en cuenta que diversos autores hablan de la heterogeneidad en la sintomatología del rechazo escolar, haciendo eco a problemáticas internalizantes emocionales, tales como la tristeza, fatiga, ansiedad, miedo, y timidez, y a problemas externalizantes de conducta tales como bajo nivel de la tolerancia, incumplimiento de normas sociales, rabietas, fugas de la escuela 0 el hogar, agresión, trastorno de ansiedad generalizada, trastorno negativista desafiante y depresión (Achenbach y Edelbrock., 1986; Kearney y Albano, 2004; Quay, 1987).

Asimismo, cabe destacar que la búsqueda de refuerzos tangibles fuera del ámbito escolar se manifiesta a consecuencia de mantener un refuerzo positivo (Ortega-Sandoval, 2019). En este caso, los refuerzos se contemplan en la ejecución de actividades con sus amigos, en ver la TV, dormir, realizar deporte 0 , más allá de ello, en el uso de drogas 0 actos delictivos, lo que puede desarrollar trastornos desafiantes y desórdenes en la conducta (Kearney y Albano, 2004; Bada, 2015). Y, estas con- 
ductas además pueden cambiar con la edad. Es decir, en el caso de los jóvenes, a diferencia de los niños, es fácil sentirse atraídos por estímulos atractivos que pueden obtener fuera de la escuela. Por lo que, la conducta de rechazo escolar en este colectivo es más crónico que los grupos que rechazan la escuela por otros motivos, y frecuentemente se vincula a conflictos 0 situaciones problemáticas en el ámbito familiar (Kearney y Silverman, 1995).

A este respecto es importante destacar que el PSP predice positiva y significativamente una alta ansiedad escolar (Vicent et al., 2014). Este desajuste psicológico puede conllevar a buscar refuerzos positivos con objeto de reducir la ansiedad que le produce la escuela.

Asimismo, se ha de tener en cuenta en un PAO-C es marcado por un afecto negativo y neuroticismo (Vicent et al., 2019), así como un mayor nivel de ansiedad, depresión y estrés (O'Connor, Rasmussen y Hawton, 2010). Es decir, se vincula con diversos desórdenes al sentir una alta autocrítica que le puede conllevar a sentimientos de interioridad y culpa por no haber cumplido los estándares marcados. Es por ello, que la búsqueda de refuerzos positivos fuera del ámbito escolar pueda ser una vía de escape para este tipo de alumnado.

No obstante, estudios anteriores marcan el PAO-S como un perfeccionismo vinculado a una mayor competencia escolar debido al esfuerzo (Liem, 2015). De hecho, el estudio realizado por Vicent et al. (2019), al contrario del PAO-C, el PAO-S se asociaba negativa y significativamente al afecto negativo y neuroticismo, y se vinculaba estrechamente a la extraversión y a la apertura a la experiencia. Se habla, por tanto, de una mayor seguridad y entusiasmo por lograr la perfección. Tal vez, hay casos en los que esa gran seguridad en sí mismo despierte en sí un sentimiento egocéntrico en el que haga sentir al individuo que incluso optando por otras vías de refuerzos positivos se pueda llegar al objetivo marcado. Asimismo, puede ser que ese mismo sentimiento decaiga, en ocasiones, hacia el aburrimiento.

No obstante, son hipótesis que deberían ser probadas en estudios con muestras similares y con distinta cultura, debido a que todo apunta que las investigaciones anteriores se asocian positivamente con las variables de ajuste de la personalidad (Vicent et al., 2019). Además, haciendo eco a Gonzálvez et al. (2018), pese el rechazo escolar vincularse con la internalización de las emociones, cabe tener en cuenta que el factor IV del SRAS-R-C contempla más aspectos externos que internos a déficits emocionales. Por ello, es importante que futuros estudios repliquen el trabajo con muestras de edad similares para observar si los hallazgos se repiten. Asimismo, se debería replicar el trabajo con muestras etarias distintas a la observada en el presente estudio con objeto de establecer diferencias y similitudes y analizar si las posibles controversias halladas entre estudios tienen que ver con la diferencia de edad.

\section{CONCLUSIONES}

En conclusión, el estudio actual muestra un avance en el conocimiento científico educativo para la sociedad ecuatoriana, haciendo eco de la necesidad de establecer estrategias de intervención y/o prevención de las distintas dimensiones de perfeccionismo para poder disminuir no solo el alto rechazo escolar producido en estos sujetos, sino todas las posibles variables de desajuste psicológico que desencadena el constructo (Burns, 1980; Flett y Hewitt, 2002; Frost, Marten, Lahart y Rosenblate, 1990; Hamacheck, 1978; Hewitt y Flett, 1991; Ortega-Sandoval, 2019; Stoeber y Stoeber, 2009; Vicent; 2017; Vicent et al., 2019).

\section{REFERENCIAS BIBLIOGRÁFICAS}

Achenbach, T. M. y Edelbrock, C. S. (1986). Teacher s Report Form. TRF. Burlington: University of Vermont.

Bada, S. 0. (2015). School Refusal Behavior: a psycho-behavioural disorder in children and adoles- 
cent. National Journal of Inclusive Education, 3(1), 241-249.

Buitelaar, J. K., van Andel, H., Duyx, J. H. M. y van Strien, D. C. (1994). Depressive and anx- iety disorders in adolescence: A follow-up study of adolescents with school refusal. Acta Paedopsychiatrica, 56, 249-253

Burns, D. D. (1980). The Perfectionist's script for self-defeat. Psychology today. 14(6), 34-52.

Cohen, J. (1988). Statistical power analysis for the behavioral sciences. Hillsdale, NJ: Erlbaum.

Egger, H. L., Costello, E. J. y Angold, A. (2003). School refusal and psychiatric disorders: A community study. Journal of the American Academy of Child and Adolescent Psychiatry, 42, 797807. doi: 10.1097/01.CHI.0000046865.56865.79

Flett, G. L. y Hewitt, P. L. (2002). Perfectionism and maladjustment: An overview of theoretical, definitional, and treatment issues. En P. L. Hewitt y G. L. Flett (Eds.), Perfectionism: Theory, research, and treatment (pp. 5-31). Washington, DC: American Psychological Association.

Flakierska, N., Lindstrom, M. y Gillberg, C. (1988). School refusal: A 15 to 20-year follow-up study of 35 Swedish urban children. The British Journal of Psychiatry, 152 (6), 834-837.

Flett, G. L., Hewitt, P. L., Besser, A., Su. C., Vaillancourt, T., Boucher, D., ... Gale, O. (2016). The child-adolescent perfectionism scale: development, psychometric of Psychoeducational Assessment, 34 (7), 634-652. doi: 10.1177/07342829166651381

Flett, G. L., Hewitt, P. L., Boucher, D. J., Davidson, L. A. y Munro, Y. (2000). The Child-Adolescent Perfectionism Scale: development, validation, and association with adjustment. Manuscrito inédito, York University, Toronto, Ontario, Canadá.

Frost, R. O., Marten, P., Lahart, C. y Rosenblate, R. (1990). The dimensions of perfectionism. Cognitive Therapy and Research, 14, 449-468.

Gonzálvez, C., Inglés, C.J., Kearney, C.A., Vicent, M., Sanmartín, R. y García-Fernández, J.M. (2016). School Refusal Assessment Scale-Revised: Factorial invariance and latent means differences across gender and age in Spanish Children. Frontiers in Psychology, 7, 1-10. doi: 10.3389/fpsyg.2016.02011

Gonzálvez, C., Inglés, C. J., Sanmartín, R., Vicent, M., Calderón, C. M. y García-Fernández, J. M. (2018). Testing factorial invariances and latent means differences of the school refusal assessment scale-revised in Ecuadorian adolescents. Current Psychology, 1-10. doi: 10.1007/s12144018-9871-1

Hamacheck, D. (1978). Psycho-dynamics of normal and neurotic perfectionism. Psychology. 15, 2733.

Heyne, D., King, N. J., Tongue, B. J. y Cooper, H. (2001). School refusal: Epidemiology and management. Pediatric Drugs, 3(10), 719-732.

Kearney, C. A. (2006). Confirmatory factor analysis of the School Refusal Assessment Scale-Revised: Child and parent versions. Journal of Psychopathology and Behavioral Assessment, 28 (3), 139-144. doi: 10.1007/s10862-005-9005-6

Kearney, C. A. (2001). School refusal behavior in youth: A functional approach to assessment and treatment. Washington, DC: American Psychological Association

Kearney, C. A. (1995). School refusal behavior. In A. R. Eisen, C. A. Kearney, \& C. E. Schaefer (Eds.), Clinical handbook of anxiety disorders in children and adolescents (pp. 19-52). Northvale, NJ: Jason Aronson.

Kearney, C. A. y Albano, A. M. (2000). When children refuse school: A cognitive-behavioral therapy approach/Therapist s guide. San Antonio, TX: The Psychological Corporation.

Kearney, C. A. y Albano, A. (2004). The functional profiles of school refusal behavior: Diagnostic aspects. Behavior Modification, 28(1), 147-161. doi: 10.1177/01454455033259263 
Hewitt, P. L. y Flett, G. L. (1991). "Perfectionism in the self and social contexts: Conceptualization, assessment, and association with psychopathology". Journal of personality and social psychology, 60 (3), 456-470.

Kearney, C. A. y Silverman, W. K. (1990). A preliminary analysis of a functional model of assessment and treatment for school refusal behavior. Behavior Modification, 14, 340-366.

Kearney, C. y Silverman, W. (1999). Functionally-based prescriptive and nonprescriptive treatment for children and adolescents with school refusal behavior. Behavior Therapy. 30(4), 673-695. doi: 10.1016/S0005-7894(99)80032-X

Last, C. G. y Strauss, C. C. (1990). School refusal in anxiety- disordered children and adolescents. Journal of the American Academic of Child and Adolescent Psychiatiry, 29(1), 31-35. doi: 10.1097/00004583-199001000-00006

Liem, G.A. (2015). Academic and social achievement goals: their additive, interactive, and specialized effects on school functioning. British Journal of Educational Psychology, 86(1), 37-56. doi: 10.1111/bjep.12085

McShane, G., Walter, G. y Rey, J. M. (2001). Characteristics of adolescents with school refusal. Australian and New Zealand Journal of Psychiatry, 35, 822-826. doi: 10.1046/j.14401614.2001.00955.x

O'Connor, R.C., Rasmussen, S. y Hawton, K. (2010). Predicting depres-sion, anxiety and self-harm in adolescents: the role of perfectionism and acute life stress. Behaviour Research and Therapy, 48, 52-59. doi: 10.1016/j.brat.2009.09.008

Ortega- Sandoval, V. N. (2019). Rechazo escolar del alumnado de Bachillerato en la ciudad de Quito (Tesis doctoral). Universidad de Alicante, Alicante, España

Quay, H. C. (1987). Patters of delinquet behavior, En H. C. Quay (Ed.), Handbook of juvenile deliquency (pp. 118-183). New York: Wiley.

Stoeber, J. y Stoeber, F. S. (2009). Domains of perfectionism: Prevalence and relationships with perfectionism, gender, age, and satisfaction with life. Personality and Individual Differences, 46, 530-535.

Vicent, M. (2017). Estudio del perfeccionismo y su relación con variables psicoeducativas en la infancia tardía [Study of perfectionism and its relationship with psycho-educational variables during late childhood] (Tesis doctoral). Universidad de Alicante, Alicante, España.

Vicent, M., Gonzálvez, C., Lagos-San Martín, N., Gomis, N., Inglés, C. J. y García-Fernández, J. M. (2014). El perfeccionismo como predictor del rechazo escolar. En Espada, Orgilés, Piqueras y Carratalá (comps.), IV Symposium nacional de psicología clínica y de la salud con niños y adolescentes (pp. 73). Madrid: Pirámide.

Vicent, M., Inglés, C. J., Sanmartín, R., Gonzálvez, C., Aparicio-Flores, M. P. y García-Fernández, J. M. (2019). Clarifying the two facets of Self-Oriented Perfectionism: influences on affect and the Big Five traits of pesonality in children. Anales de psicología, 35 (2), 280-289. doi: 10.6018/anales.35.2.331441 
